\title{
Growth-Differentiation Factor-15 as Additional Prognostic Biomarkers in Heart Failure
}

\section{Berezin $\mathrm{AE}^{*}$}

Internal Medicine Department, State Medical University of Zaporozhye, Zaporozhye, Ukraine

"Correspondence author: Berezin AE, Professor, MD, PhD, Private Clinic "Vita-Center", Senior Consultant of Therapeutic Unit, Internal Medicine Department, State Medical University of Zaporozhye, 3, Sedova Str., Zaporozhye, Ukraine, Tel: +380612894585; E-mail: dr_berezin@mail.ru; aeberezin@gmail.com

Received date: August 10, 2017; Accepted date: August 22, 2017; Published date: August 28, 2017

Copyright: ( 2017 Berezin AE. This is an open-access article distributed under the terms of the Creative Commons Attribution License, which permits unrestricted use, distribution, and reproduction in any medium, provided the original author and source are credited.

\begin{abstract}
Heart Failure (HF) remains to be a leading factor of cardiovascular morbidity and mortality. Although risk stratification of HF is promising prediction care, there are several controversies regarding choosing more optimal combinations of biomarkers and method (single versus serial measurements) of biomarker use in routine clinical practice. Growth differentiation factor-15 (GDF-15) is considered a biomarker associated with cardiac/vascular remodeling, oxidative stress, fibrosis and inflammation that were proposed to stratify HF patients at risk of death. It has been suggested that GDF-15 adding to natriuretic peptides or other conventional biomarkers (soluble ST2, cardiac troponins and galectin-3) might improve discriminative value of entire predictive models. The short communication is depicted the discussion about the perspectives of clinical use of GDF-15 in risk stratification of HF.
\end{abstract}

Keywords: Heart Failure (HF); Biomarkers; Growth differentiation factor-15; Prediction

\section{Introduction}

Heart Failure (HF) is reported as a leading cause of premature Cardiovascular $(\mathrm{CV})$ death and increased hospitalization rate in patients with established CV disease [1,2]. Although frequency of newly diagnosed HF in developed countries exhibits a trend to decrease, there is evidence regarding steadily growth of HF patients' population worldwide [3]. Biological markers are widely used to stratify individuals at higher risk of $\mathrm{HF}$ and diagnose of asymptomatic and symptomatic cardiac dysfunction regardless its etiology [4,5]. Moreover, biomarker target therapy of HF is considered as promising strategy to improve clinical outcomes amongst HF patients, while its role is not still confirmed [6]. According to contemporary Scientific Statement from the American Heart Association regarding use of biomarkers in HF only Natriuretic Peptides (NPs), cardiac troponins, galectin-3 and soluble ST2 receptors (sST2) have validated to clinical targets mentioned above [6]. However, there is a large body of evidence regarding several limitations in biomarker approaches especially in predicting incident of $\mathrm{CV}$ events and $\mathrm{CV}$ mortality among asymptomatic individuals from the general population, beyond traditional CV risk factors including diabetes mellitus, kidney disease, age, as well as medication use and conventional echocardiographic/ other images measures [7,8]. In this context, the discovery and validation of novel biomarkers or multiple biomarker strategy approaches that could improve predictive abilities of conventional biomarkers such as NPs in HF risk stratification appear to be fairly promising.

Growth differentiation factor-15 (GDF-15) is considered a biomarker associated with cardiac/vascular remodeling, oxidative stress, fibrosis and inflammation that were proposed to stratify HF patients at risk of death [9]. In physiological states GDF-15 is secreted by cardiac myocytes and it regulated growth and proliferation of tissues located in heart and vessels [10]. Therefore, GDF-15 is realized by cardiac cells due to fluid retention and pressure overload, as well as it produced in resulting in inflammation and cell-to-cell cooperation [11].

Recent clinical studies have shown that plasma levels of GDF-15 have sufficiently increased in acute myocardial infarction [12], diabetes mellitus [13], acute and chronic HF regardless its etiology [14,15], as well as independently predicted long-term all-cause mortality and CV events even after adjusting for age, gender, kidney clearance, traditional CV risk factors and other biomarkers, such as NPs, cardiac troponins, heart-type fatty acid-binding protein, $\mathrm{sST} 2$, high-sensitivity C-reactive protein and galectin-3 [9,16-19]. Interestingly, GDF-15 is able to enhance prognostication of NPs beyond traditional CV risk factor, and echocardiography parameters in individuals without known CV disease [12] and acute/chronic HF [20].

However, there are several controversies regarding the abilities of GDF-15 to improve prognostication of conventional biomarkers in HF. First controversy relates to the fact that maximizing discriminatory accuracy of repeat measurements of GDF-15 added to NPs in HF was superior to single measurement [21]. Second controversy associated with clinical evidence of advantages of individually adjusted multiple marker approaches in provision of the greatest prognostic improvement among patients with various HF phenotypes [22,23]. Although GDF-15 was useful to detect prevalent of any HF phenotype in addition to NPs, the discriminative value of NPs, GDF-15, sST2, galectin-3 and cardiac troponins in general population was similar $[24,25]$. Third controversy is follow: In obese and diabetes mellitus subjects GDF-15 was the best predictor for all-cause mortality to NPs, while discriminative value for both markers in combination was not better than single biomarker use [26]. Finally, there was no evidence that GDF-15 had sufficiently improved treatment options of HF in single and serial measures [27]. However, more large clinical studies require explaining the advantages of GDF-15 in multiple biomarker strategy in $\mathrm{HF}$. 


\section{Conclusion}

In conclusion, multiple biomarker strategies biased on NPs, sST2, cardiac troponins and probably GDF-15 are superior than single biomarker measure in prediction of $\mathrm{HF}$, as well as serial measures versus single determination of only biomarker are superior to optimize HF patient management. However, individualized biomarker approaches remains to be fairly personally adjusted to be adequately assayed and prospectively assessed in long-term period. Although GDF-15 appears to be promising biomarker in HF risk stratification, its role in prognostication requires to be additionally confirmed in large clinical trials.

\section{References}

1. Ponikowski P, Voors AA, Anker SD, Bueno H, Cleland JG, et al. (2016) Authors/Task Force Members. 2016 ESC guidelines for the diagnosis and treatment of acute and chronic heart failure: the Task Force for the diagnosis and treatment of acute and chronic heart failure of the European Society of Cardiology (ESC) developed with the special contribution of the Heart Failure Association (HFA) of the ESC. Eur Heart J 37: 2129-2200.

2. Yancy CW, Jessup M, Bozkurt B, Butler J, Casey DE, et al. (2017) ACC/AHA/HFSA Focused Update of the 2013 ACCF/AHA Guideline for the Management of Heart Failure: A Report of the American College of Cardiology/American Heart Association Task Force on Clinical Practice Guidelines and the Heart Failure Society of America. J Card Fail.

3. Mozaffarian D, Benjamin EJ, Go AS, Arnett DK, Blaha MJ, et al. (2016) American Heart Association Statistics Committee; stroke statistics subcommittee. Heart Disease and Stroke Statistics-2016 Update: A Report From the American Heart Association. Circulation 133: e38-e360.

4. Van den Berg MJ, Bhatt DL, Kappelle LJ, de Borst GJ, Cramer MJ, et al. (2017) SMART study group; REACH Registry investigators. Identification of vascular patients at very high risk for recurrent cardiovascular events: validation of the current ACC/AHA very high risk criteria. Eur Heart J.

5. Pouleur AC (2015) Which biomarkers do clinicians need for diagnosis and management of heart failure with reduced ejection fraction? Clin Chim Acta 443: 9-16.

6. Chow SL, Maisel AS, Anand I, Bozkurt B, de Boer RA, et al. (2017) American Heart Association Clinical Pharmacology Committee of the Council on Clinical Cardiology; Council on Basic Cardiovascular Sciences; Council on Cardiovascular Disease in the Young; Council on Cardiovascular and Stroke Nursing; Council on Cardiopulmonary, Critical Care, Perioperative and Resuscitation; Council on Epidemiology and Prevention; Council on Functional Genomics and Translational Biology; and Council on Quality of Care and Outcomes Research. Role of Biomarkers for the Prevention, Assessment, and Management of Heart Failure: A Scientific Statement From the American Heart Association. Circulation.

7. Wettersten N, Maisel AS (2016) Biomarkers for heart failure: an update for practitioners of internal medicine. Am J Med 129: 560-567.

8. Berezin AE (2016) Prognostication in different heart failure phenotypes: the role of circulating biomarkers. Journal of Circulating Biomarkers 5: 1 .

9. Pareek M, Bhatt DL, Vaduganathan M, Biering-Sørensen T, Qamar A, et al. (2017) Single and multiple cardiovascular biomarkers in subjects without a previous cardiovascular event. Eur J Prev Cardiol.

10. Wang T, Liu J, McDonald C, Lupino K, Zhai X, et al. (2017) GDF15 is a heart-derived hormone that regulates body growth. EMBO Mol Med 9: 1150-1164.
11. Zankov DP, Sato A, Shimizu A, Ogita H (2017) Differential effects of myocardial afadin on pressure overload-induced compensated cardiac hypertrophy. Circ J.

12. Berezin AE (2016) Diabetes mellitus related biomarker: The predictive role of growth-differentiation factor-15. Diabetes Metab Syndr 10: S154S157.

13. Berezin A (2016) Biomarkers for cardiovascular risk in diabetic patients. Heart 102: 1939-1941.

14. Berezin A, Kremzer A, Martovitskaya Y, Samura T, Berezina T (2016) The novel biomarker risk prediction score in patients with chronic heart failure. Clinical Hypertension 22.

15. Lichtenauer M, Jirak P, Wernly B, Paar V, Rohm I, et al. (2017) A comparative analysis of novel cardiovascular biomarkers in patients with chronic heart failure. Eur J Intern Med.

16. Skau E, Henriksen E, Wagner P, Hedberg P, Siegbahn A, et al. (2017) GDF-15 and TRAIL-R2 are powerful predictors of long-term mortality in patients with acute myocardial infarction. Eur J Prev Cardiol.

17. Schernthaner C, Lichtenauer M, Wernly B, Paar V, Pistulli R, et al. (2017) Multibiomarker analysis in patients with acute myocardial infarction. Eur J Clin Invest.

18. Berezin AE, Kremzer AA (2014) Relationship between circulating endothelial progenitor cells and insulin resistance in non-diabetic patients with ischemic chronic heart failure. Diabetes \& Metabolic Syndrome: Clinical Research \& Reviews 8: 138-144.

19. Wang H, Chen Q, Li Y, Jing X, Yang J (2017) Prognostic value of growth differentiation factor-15 in Chinese patients with heart failure: A prospective observational study. Cardiol J.

20. Demissei BG, Cotter G, Prescott MF, Felker GM, Filippatos G, et al. (2017) A multimarker multi-time point-based risk stratification strategy in acute heart failure: results from the RELAX-AHF trial. Eur J Heart Fail 19: 1001-1010.

21. Hage C, Michaëlsson E, Linde C, Donal E, Daubert JC, et al. (2017) Inflammatory biomarkers predict heart failure severity and prognosis in patients with heart failure with preserved ejection fraction: a holistic proteomic approach. Circ Cardiovasc Genet 10: e001633.

22. Berezin AE (2015) Biological markers of cardiovascular diseases. Part 3 Diagnostic and prognostic value of biological markers in stratification of patient $\mathrm{s}$ with cardiometabolic risk. Lambert Academic Publishing $\mathrm{GmbH}$, Moscow.

23. Sinning C, Kempf T, Schwarzl M, Lanfermann S, Ojeda F, et al. (2017) Biomarkers for characterization of heart failure - Distinction of heart failure with preserved and reduced ejection fraction. Int J Cardiol 227: 272-277.

24. Sinning C, Ojeda F, Wild PS, Schnabel RB, Schwarzl M, et al. (2017) Midregional proadrenomedullin and growth differentiation factor-15 are not influenced by obesity in heart failure patients. Clin Res Cardiol 106: 401-410.

25. Baggen VJ, van den Bosch AE, Eindhoven JA, Schut AW, Cuypers JA, et al. (2017) Prognostic value of N-Terminal Pro-B-Type Natriuretic Peptide, Troponin-T, and Growth-Differentiation Factor 15 in Adult Congenital Heart Disease. Circulation 135: 264-279.

26. Berezin A (2017) Up-to-date clinical approaches of biomarkers' use in heart failure. Biomed Res Ther 4: 1341-1370.

27. Berezin A (2017) Contemporary approaches of biological markers in heart failure. Scholars' Press, OmniScriptum Management $\mathrm{GmbH}$, Saarbrücken, Germany. 